\title{
Sarcoidosis with major airway, vascular and nerve compromise
}

\author{
Hiroshi Sekiguchi MD, Jun Suzuki, James P Utz MD
}

\begin{abstract}
H Sekiguchi, J Suzuki, JP Utz. Sarcoidosis with major airway, vascular and nerve compromise. Can Respir J 2013;20(4): 256-258.

The present report describes a 60-year-old Caucasian woman who presented with progressive dyspnea, cough and wheeze. A computed tomography scan of the chest showed innumerable bilateral inflammatory pulmonary nodules with bronchovascular distribution and a mediastinal and hilar infiltrative process with calcified lymphadenopathy leading to narrowing of lobar bronchi and pulmonary arteries. An echocardiogram revealed pulmonary hypertension. Bronchoscopy showed left vocal cord paralysis and significant narrowing of the bilateral bronchi with mucosal thickening and multiple nodules. Transbronchial biopsy was compatible with sarcoidosis. Despite balloon angioplasty of the left lower lobe and pulmonary artery, and medical therapy with oral corticosteroids, her symptoms did not significantly improve. To the authors' knowledge, the present report describes the first case of pulmonary sarcoidosis resulting in major airway, vascular and nerve compromise due to compressive lymphadenopathy and suspected concurrent granulomatous infiltration. Its presentation mimicked idiopathic mediastinal fibrosis.
\end{abstract}

Key Words: Airway narrowing; Mediastinal fibrosis; Pulmonary hypertension; Sarcoidosis; Vocal cord paralysis

Learning objectives:

1. To recognize various mechanisms of dyspnea caused by intrathoracic sarcoidosis.

2. To understand the pathophysiology of sarcoidosis-associated pulmonary hypertension (SAPH).

CanMEDS competency: Medical Expert

Pre-test:

1. What are the proposed mechanisms of sarcoidosis-induced central airway narrowing or bronchial stenosis?

2. What are the proposed mechanisms of SAPH?

\section{CASE PRESENTATION}

A 60 -year-old Caucasian female nonsmoker presented with a fiveyear history of progressive exertional dyspnea associated with chronic cough. Examination revealed moderate dysphonia and a highpitched monophonic wheeze in the anterior chest. The pulmonic component of the second heart sound was intensified, with no third right ventricular heart sound. There was no sign of decompensated right ventricular failure in the physical examination. The patient denied chest pain, palpitation or syncopal episode. She did not experience symptoms or recent changes in her skin, vision, gastrointestinal or musculoskeletal systems. Social, occupational and family history were unremarkable.

Laboratory testing revealed normal complete blood cell counts, electrolyte levels, liver function tests, fungal serologies, QuantiFERON-TB Gold (Cellestis Ltd, Australia) and angiotensin-converting enzyme level.

\section{Une sarcoïdose pulmonaire responsable d'une atteinte importante des voies aériennes et des structures vasculaires et nerveuses}

Le présent rapport décrit le cas d'une femme blanche de 60 ans qui a consulté en raison d'une dyspnée progressive, d'une toux et d'une respiration sifflante. La tomodensitométrie du thorax a révélé d'innombrables nodules pulmonaires inflammatoires bilatéraux en distribution bronchovasculaire et un processus infiltratoire du médiastin et de l'hile accompagné d'une lymphadénopathie calcifiée ayant entraîné un rétrécissement des bronches lobaires et des artères pulmonaires. Un échocardiogramme a révélé une hypertension pulmonaire, tandis que la bronchoscopie a exposé une paralysie des cordes vocales gauches et un important rétrécissement des bronches bilatérales avec un épaississement muqueux et de multiples nodules. La biopsie transbronchique était compatible avec une sarcoïdose. Malgré une angioplastie à ballonnet du lobe inférieur gauche et de l'artère pulmonaire et une médicothérapie aux corticoïdes par voie orale, ses symptômes ne se sont pas vraiment atténués. En autant que le sache l'auteur, le présent rapport décrit le premier cas de sarcoïdose pulmonaire responsable d'une atteinte importante des voies aériennes et des structures vasculaires et nerveuses en raison d'une lymphadénopathie de compression et d'une infiltration granulomateuse concomitante présumée. Sa présentation imite celle d'une fibrose idiopathique du médiastin.

Pulmonary function testing showed mild obstruction (forced expiratory volume in $1 \mathrm{~s}\left[\mathrm{FEV}_{1}\right] 64 \%$ of predicted) with reduced diffusing capacity for carbon monoxide (DLCO, $56 \%$ predicted). An echocardiogram revealed normal left ventricular systolic function with no regional wall motion abnormality and mild right ventricular enlargement with pulmonary hypertension (estimated right ventricular systolic pressure $54 \mathrm{mmHg}$ ). A computed tomography (CT) scan of the chest showed innumerable bilateral inflammatory pulmonary nodules with a bronchovascular distribution, and a mediastinal and hilar infiltrative process with calcified lymphadenopathy (especially stations 10 , 11 and 12) leading to narrowing of lobar bronchi and pulmonary arteries (Figure 1). A ventilation/perfusion lung scan demonstrated significantly decreased perfusion to the left lung (11\%) and equal lung ventilation bilaterally.

Differential diagnosis included idiopathic mediastinal fibrosis, diffuse granulomatous disease and infection. Bronchoscopy showed significant narrowing of the bilateral bronchi with mucosal thickening and multiple nodules. Attempts to biopsy bronchial mucosa caused significant bleeding and the procedure was halted. Bronchial washings returned negative for bacterial, mycobacterial and fungal culture. Right heart catheterization with pulmonary angiogram was performed to assess pulmonary hypertension and the perfusion defect in the ventilation/perfusion scan. It demonstrated a mean pulmonary arterial pressure of $26 \mathrm{mmHg}$ with stenosis of bilateral pulmonary arteries, especially the left lower lobe pulmonary artery. Balloon angioplasty of the left lower lobe pulmonary artery was performed with mild improvement in calibre (Figure 2). A repeat angiogram was scheduled for further dilation and possible stent placement.

The patient returned to the clinic two months later with worsening hoarseness and no improvement of her dyspnea. A CT scan of the

Division of Pulmonary and Critical Care Medicine, Mayo Clinic, Rochester, Minnesota, USA

Correspondence: Dr Hiroshi Sekiguchi, Division of Pulmonary and Critical Care Medicine, Mayo Clinic, 200 First Street Southwest, Rochester,

Minnesota 55905, USA. Telephone 507-284-2416, fax 507-266-4372, e-mail sekiguchi.hiroshi@mayo.edu 


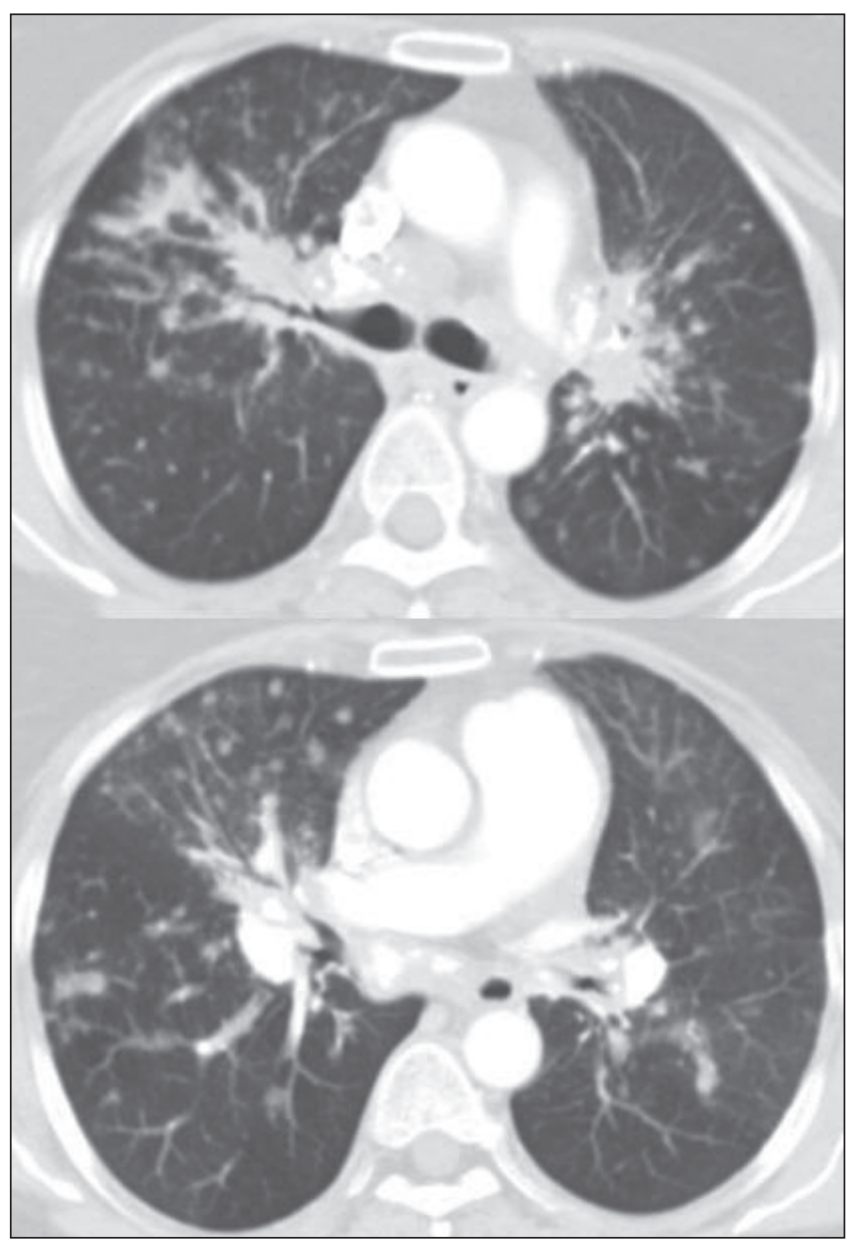

Figure 1) Computed tomography scan of the chest demonstrating innumerable bilateral pulmonary nodules with a bronchovascular distribution, marked lymphadenopathy, narrowing of lobar bronchi and compression of left pulmonary arteries

chest was essentially unchanged. She declined pulmonary angioplasty. A repeat bronchoscopy showed left vocal cord paralysis and similar abnormalities noted in the previous bronchoscopy. Microbiology on bronchial washing returned normal. Transbronchial biopsy of the right upper and lower middle lobes showed non-necrotizing granulomatous inflammation. Special stains were negative for micoorganisms. The morphological features were compatible with sarcoidosis (Figure 3). The patient was started on prednisone $40 \mathrm{mg}$ daily. Her dyspnea and hoarseness partially improved and the prednisone dosage was gradually decreased. Unfortunately, her symptoms did not significantly improve with two years of corticosteroid therapy. Repeat pulmonary function testing demonstrated mild obstruction $\left(\mathrm{FEV}_{1} 61 \%\right.$ predicted) and decrease in diffusing capacity (DLCO 58\% predicated). A ventilation/ perfusion lung scan showed slightly improved perfusion to the left lung (27\%). Hydroxychloroquine was added to the corticosteroid therapy.

\section{DISCUSSION}

Sarcoidosis is a multisystem idiopathic granulomatous disease of unknown etiology. Granuloma in sarcoidosis is characterized by epithelioid histiocytes and multinucleate giant cells with interspersed $\mathrm{CD}^{+}$lymphocytes (1). Pulmonary manifestation is very common and the lungs are affected in $>90 \%$ of patients with sarcoidosis (1). In our case, the patient's respiratory symptoms were attributed to multiple mechanisms.

First, diffuse granulomatous inflammation and narrowing of the major bronchi were considered to be major contributors to her cough and dyspnea. Inflammation of the airway mucosa presents as mucosal

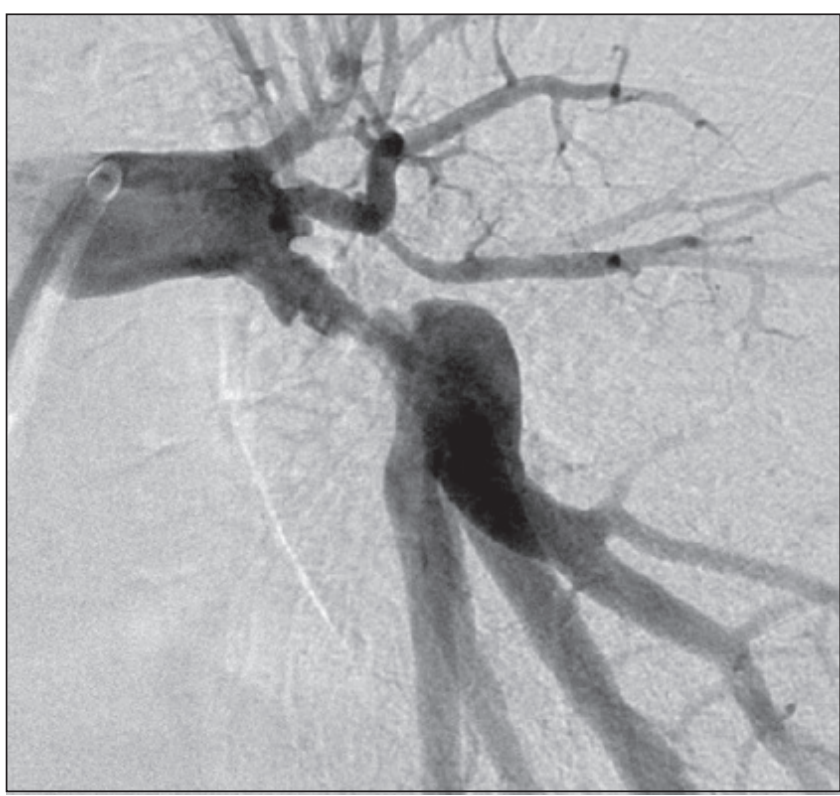

Figure 2) Pulmonary artery angiogram demonstrating narrowing of the left lower lobe pulmonary artery

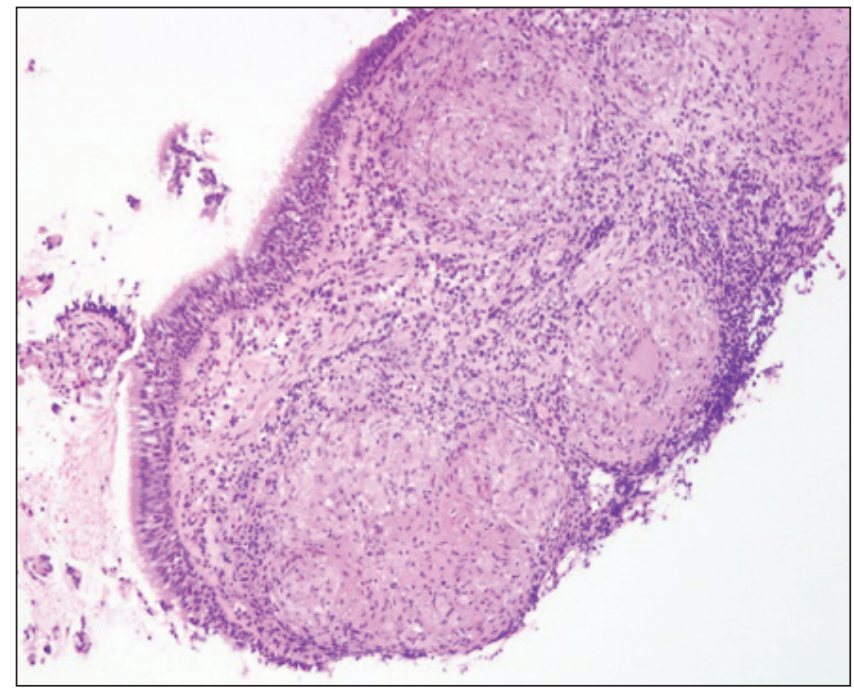

Figure 3) Transbronchial biopsy showing well-formed granulomas typical of sarcoidosis in the airway wall

edema, erythema and granulomatous nodule formation. Cough is usually the main symptom; inflammation itself seldom produces significant obstructive or airway dysfunction (2). Although major airway compression or narrowing due to sarcoidosis is uncommon, it can cause disabling inspiratory and expiratory airflow limitation (3). A previous study reported only 18 of 2500 patients with sarcoidosis demonstrating bronchial stenosis (3). Proposed mechanisms include the direct pressure or spread of granulomatous process from the enlarged lymph nodes to the bronchi, in addition to the endobronchial occlusion caused by mucosal inflammation and sarcoid granulomas spreading in the submucosa (3). These two mechanisms are not mutually exclusive and can occur simultaneously (3), as demonstrated in our case with CT scan and bronchoscopy.

Second, compression of pulmonary arteries due to pulmonary hypertension and subsequent perfusion mismatch likely had a significant impact on her respiratory status. Previous studies have reported a prevalence of SAPH ranging from $6 \%$ to $28 \%$, and is higher in advanced sarcoidosis requiring lung transplantation. Proposed mechanisms include granulomatous arteriovenopathy, pulmonary veno-occlusive disease, 
external compression of the pulmonary vessels by enlarged mediastinal lymph nodes or fibrosis, hypoxemia-induced vasoconstriction, pulmonary vascular remodelling and myocardial dysfunction (4). In the present case, no findings suggestive of granulomatous arteriovenopathy, venous occlusion or vascular remodelling were detected on the transbronchial biopsy specimen and no myocardial dysfunction was apparent in echocardiography. Therefore, external compression of the pulmonary vessels was deemed to be a major cause of pulmonary hypertension. Although a balloon angioplasty of the pulmonary artery was not successful in the present case, a previous report of two cases described successful stenting and hemodynamic improvement in pulmonary artery stenosis due to sarcoidosis-related mediastinal fibrosis (5). Both patients were diagnosed with stage 1 pulmonary sarcoidosis and later presented with pulmonary hypertension secondary to external compression of the pulmonary arteries by enlarged lymphadenopathy. While corticosteroids are commonly used, treatment strategies for SAPH have not yet been established. One case series indicated that a trial with epoprostenol improved the pulmonary vascular resistance and WHO classification in patients with SAPH (6).

Third, left vocal cord paralysis resulted in hoarseness and may have contributed to dyspnea on exertion. Approximately 10 cases of vocal cord paralysis due to sarcoidosis have been reported in the literature. They are caused by compressive lymphadenopathy with or without polyneuritis (7). The majority of these cases were unilateral left vocal cord paralysis, likely because of the unique intrathoracic course of the left recurrent laryngeal nerve as it passes around the arch of the aorta where the pathological lymphadenopathy is frequently located, as demonstrated in our case. However, bilateral vocal cord paralysis has been reported $(7,8)$.

\section{REFERENCES}

1. Baughman RP, Culver DA, Judson MA. A concise review of pulmonary sarcoidosis. Am J Respir Crit Care Med 2011;183:573-81.

2. Polychronopoulos VS, Prakash UBS. Airway involvement in sarcoidosis. Chest 2009;136:1371-80.

3. Chambellan A, Turbie P, Nunes H, Brauner M, Battesti J-P, Valeyre D. Endoluminal stenosis of proximal bronchi in sarcoidosis:

Bronchoscopy, function, and evolution. Chest 2005;127:472-81.

4. Palmero V, Sulica R. Sarcoidosis-associated pulmonary hypertension: Assessment and management. Semin Respir Crit Care Med 2010;31:494-500.

5. Hamilton-Craig CR, Slaughter R, McNeil K, Kermeen F, Walters DL. Improvement after angioplasty and stenting of pulmonary arteries due to sarcoid mediastinal fibrosis. Heart Lung Circ 2009;18: 222-5.

\section{CONCLUSIONS}

To our knowledge, the present report describes the first case of pulmonary sarcoidosis resulting in major airway, vascular and nerve compromise due to compressive lymphadenopathy and suspected concurrent granulomatous infiltration. Its presentation mimicked idiopathic mediastinal fibrosis. Sarcoidosis can affect major intrathoracic structures and cause a significant decline in a patient's respiratory status.

\section{Post-test}

1. What are the proposed mechanisms of sarcoidosis-induced central airway narrowing or bronchial stenosis?

Proposed mechanisms include the direct pressure (external compression) or spread of granulomatous process from the enlarged lymph nodes to the bronchi as well as endobronchial occlusion caused by mucosal inflammation and sarcoid granulomas spreading in the submucosa. These two mechanisms are not mutually exclusive and can occur simultaneously.

2. What are the proposed mechanisms of SAPH?

Previous studies have reported a prevalence of SAPH ranging from $6 \%$ to $28 \%$. Proposed mechanisms include granulomatous arteriovenopathy, pulmonary veno-occlusive disease, external compression of the pulmonary vessels by enlarged mediastinal lymph nodes or fibrosis, hypoxemia-induced vasoconstriction, pulmonary vascular remodelling and myocardial dysfunction.

DISCLOSURES: The authors have no financial disclosures or conflicts of interest to declare.

6. Fisher KA, Serlin DM, Wilson KC, Walter RE, Berman JS, Farber HW. Sarcoidosis-associated pulmonary hypertension: Outcome with long-term epoprostenol treatment. Chest 2006;130:1481-8.

7. Coffey CS, Vallejo SL, Farrar EK, Judson MA, Halstead LA. Sarcoidosis presenting as bilateral vocal cord paralysis from bilateral compression of the recurrent laryngeal nerves from thoracic adenopathy. J Voice 2009;23:631-4.

8. Witt RL. Sarcoidosis presenting as bilateral vocal fold paralysis. J Voice 2003;17:265-8. 


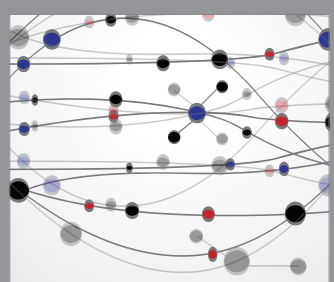

The Scientific World Journal
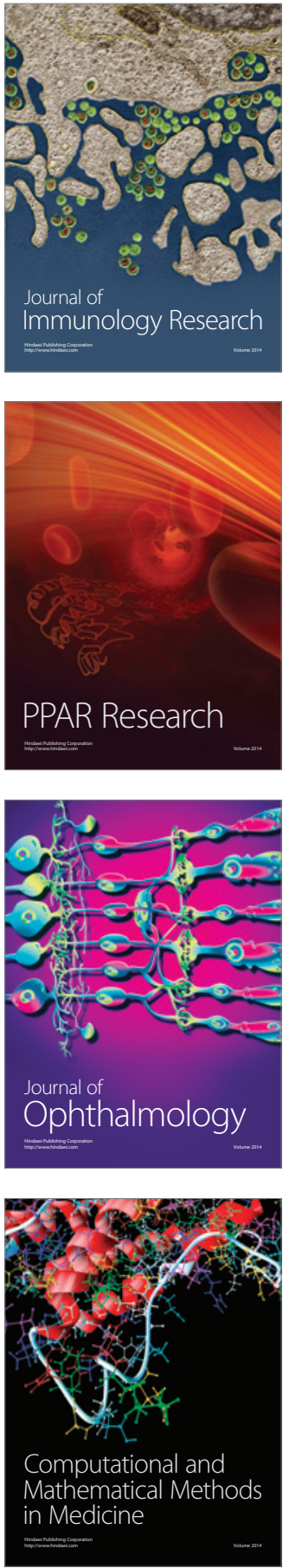

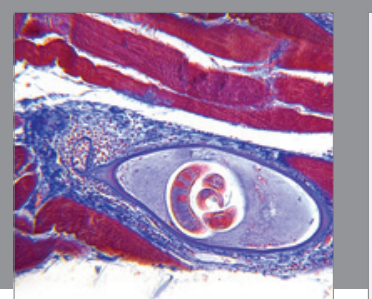

Gastroenterology Research and Practice

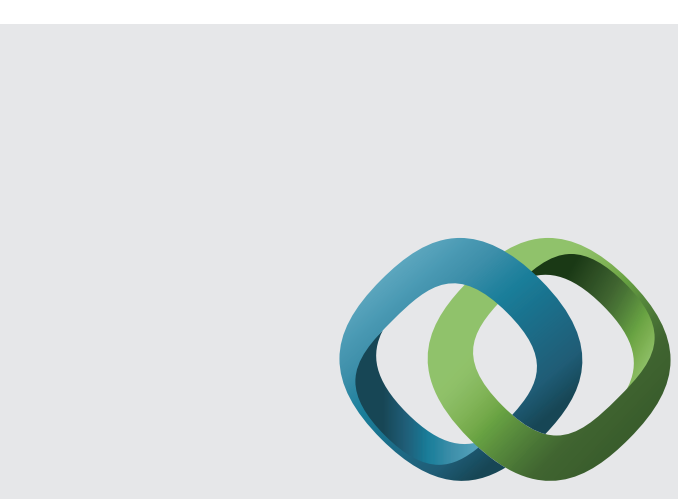

\section{Hindawi}

Submit your manuscripts at

http://www.hindawi.com
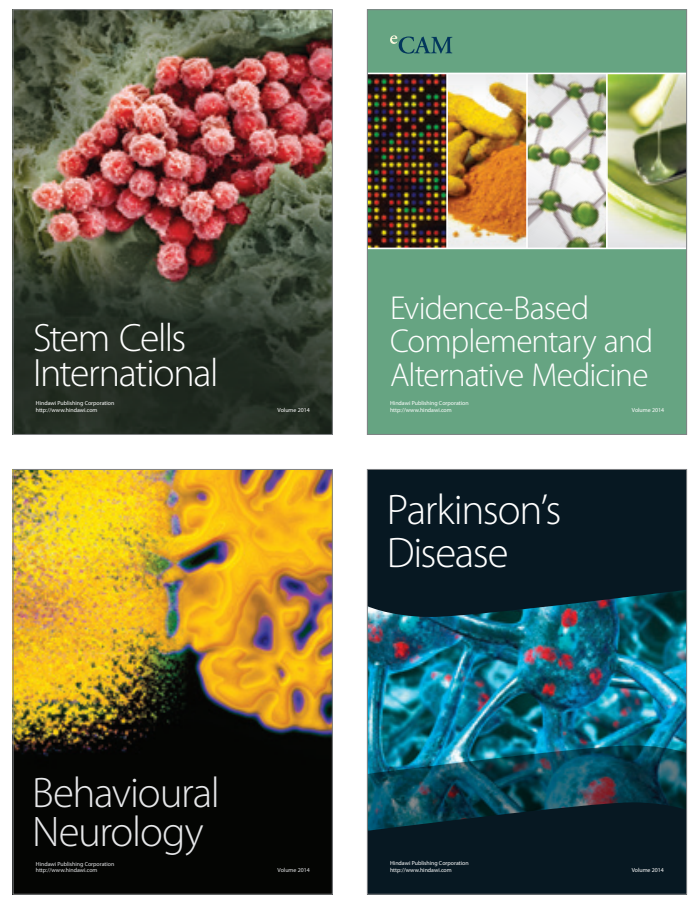
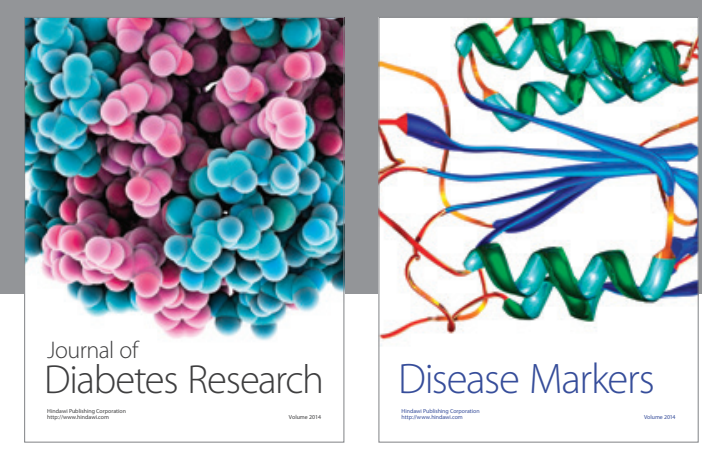

Disease Markers
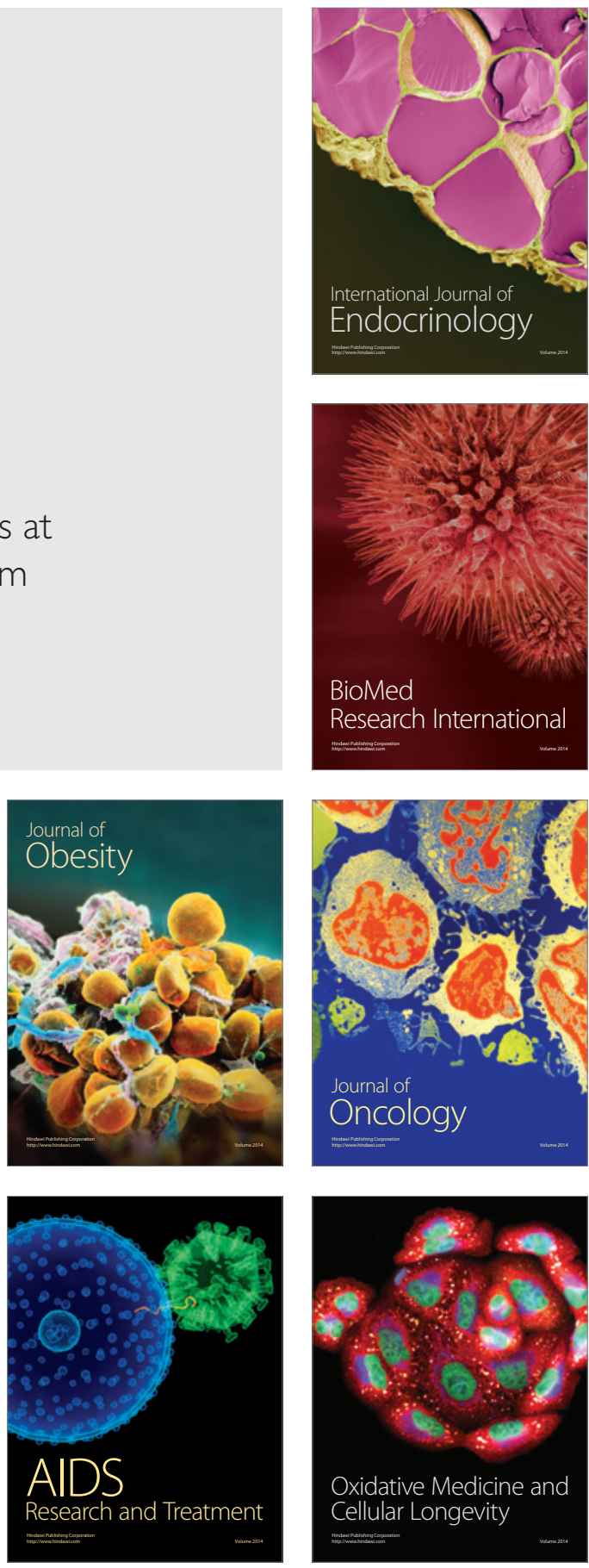